\title{
Congestion Aware and Adaptive Dynamic Source Routing Algorithm with Load-balancing in MANETs
}

\author{
A. Valarmathi \\ Assistant Professor \\ Department of Computer Applications \\ Anna University Tiruchirappalli, INDIA
}

\author{
RM. Chandrasekaran \\ Professor \\ Department of Computer Science and Engineering \\ Annamalai University, Chidambaram, INDIA
}

\begin{abstract}
The explosive growth in the use of real-time multimedia applications in mobile devices requires quality of service (QoS) support for effective communication. Network congestion is the main reason for packet loss, longer delay and jitter in streaming multimedia applications. Most of the present routing protocols are not designed to adapt congestion. In this paper, the original DSR protocol is modified to define the occurrence of congestion by monitoring and reporting multiple resource utilization thresholds as QoS attributes and use multi-path-routing and load-balancing during the periods of congestion to improve QoS in MANETs for CBR multimedia applications. NS-2 simulation was extensively carried out to evaluate the performance of the modified DSR in terms of throughput, jitter and end-to-end delay. The results were compared with the original DSR in random node movements under varying traffic loads of 10,15 and $20 \mathrm{CBR}$ traffic flows to completely assess the applicability of the modified DSR. Simulation results showed that the proposed protocol can significantly improve the network performance for low and medium traffic load in multimedia based mobile ad hoc networks by extending the network lifetime and providing fewer packet losses than the original DSR.
\end{abstract}

Keywords: Mobile ad hoc network; DSR; Congestion; Multi-path routing; Traffic load

\section{INTRODUCTION}

The frequent and unpredictable topological changes in MANET render the task of routing a challenging task and MANET inherently depends on the routing scheme employed to provide expected Quality of Service (QoS). Routing protocols for Ad hoc networks can be broadly classified into two categories as proactive and reactive. Proactive (or) table-driven routing algorithms employ distance vector based or link state based routing strategies. However, the main drawback of these algorithms is that the need for frequent table updation consumes significant amount of memory, bandwidth and battery power [1]. Example of such protocols is Optimized Link State Routing (OLSR) [2] and Destination Sequenced Distance Vector routing (DSDV) [3]. In reactive routing protocols, a node searches and maintains a route to another node only when it requires transmitting data to that particular node. This on-demand reactive approach minimizes bandwidth utilization compared to proactive routing protocols. Ad-hoc On-Demand Distance Vector (AODV) [4], Dynamic Source Routing (DSR) [5], Temporally Ordered Routing Algorithm (TORA) [6] and Associatively-Based Routing (ABR) [7] are examples of such protocols. Our proposed protocol is based on DSR. It operates on two mechanisms such as route discovery and route maintenance. All these protocols rely on single-path routing algorithms, where all traffic to a destination is required to be routed through a single successor and when a link become congested, the entire traffic has to be rerouted. Routing protocols for MANET can also categorized as congestion-unadaptive routing and congestion adaptive routing. Existing routing protocols belong to the first group where congestion nonadaptiveness in routing may result in longer delay, higher overhead and many packet losses for traffic intensive data like multimedia applications. DSR does not make dynamic routing adjustments based on memory, delay, number of service requests or battery utilization information which often indicates certain levels of usage and possible occurrences of congestion. Detection of undesired paths enables the ability to implement route admission control and to perform multi-path routing scheme which could further improve the performance of the network in a significant manner.

In the present paper, we propose a modified DSR protocol to monitor congestion or multiple resource utilization levels, notify the routing process of congestion and invoke multi-path routing during the periods of congestion for streaming multimedia applications. In addition, this paper also determine the effects of various traffic loads(low, medium, high) models on the modified routing protocol and compare the performance metrics of throughput, jitter and end-to-end delay of original DSR protocol with modified DSR protocol.

\section{RELATED WORKS}

The research on congestion for MANET is still in the early stage and there is a need for new techniques. In this section, the research work related to congestion control and multi-path routing is presented. Round trip time measurements are used to distribute load between paths in Multi-path Source Routing (MSR) [8]. A distributed multi-path DSR protocol (MP-DSR) was developed to improve QoS with respect to end-to-end reliability [9]. The protocol forwards outgoing packets along multiple paths that are subjected to an end-to-end reliability model. Split Multi-path Routing (SMR) utilized multiple routes of maximally disjoint paths which minimize route recovery process and control message overhead [10-11]. The protocol uses a per-packet allocation scheme to distribute data packets into multiple paths of active sessions which prevents nodes from being congested in heavily loaded traffic situations. Asis Nasipuri et al [12] showed how intelligent use of multi-path technique in DSR protocol can reduce the frequency of query floods. They also developed an analytic modeling framework to determine the relative frequency of query floods for various techniques. Lianfang Zhang et al [13] established a network queuing model in MSR that could incorporate cross-traffic among multiple paths. The load balancing was considered as an optimization problem and the results are verified with the existing model. Jinglun Shi et al [14] presented an independent stability-based multi-path routing 
scheme that is based on DSR. The stable multi-path embedded in the routing policies is expected to enhance the performance by decreasing rerouting overheads. Zafar Taha and Xian Liu [15] demonstrated how the use of multi-path DSR can reduce the frequency of route discovery process. The data traffic was characterized through compound Log-normal distribution. Stephen Mueller et al [16] specifically examined the issues of multi-path routing in MANETs. They indicated that most of the protocols proposed can only provide QoS in terms of specific metrics, such as bandwidth, delay or reliability. However, it may be necessary to develop mechanisms to support QoS in terms of multiple metrics. Subramanian et al [17] proposed a Multi-path Power Sensitive Routing Protocol (MPSR) based on DSR and showed how an efficient heuristic based multi-path technique can improve the mean time to node failure and maintain the variance in the power of all the nodes as low as possible. A dynamic loadaware based load-balanced routing (DLBL) algorithm was developed which considers intermediate node routing load as the primary route selection metric [18]. This helps the protocol to discover a route with less network congestion and bottlenecks. When a link breaks because of the node mobility or power off, DLBL provides efficient path maintenance to patch up broken links to help to get a robust route from the source to the destination. De Rango et al [19] proposed an energy aware multipath routing protocol by considering minimum drain rate as a metric. An update mechanism and a simple data packet scheduling among the energy efficient paths have also been implemented to update the source route cache and for improving the traffic and energy load balancing. Raghavandra et al [20] proposed Congestion Adaptive Routing in Mobile Ad Hoc Networks (CRP). In CRP every node appearing on a route warns its previous node when prone to be congested. The previous node then uses a "bypass" route bypassing the potential congestion to the first noncongested node on the route. Traffic will be split probabilistically over these two routes, primary and bypass, thus effectively lessening the chance of congestion occurrence.

The different multi-path schemes proposed earlier concentrates few of the performance aspects. For example, the measurement of jitter is very scarce, which is a critical performance metric for multimedia traffic. In addition to that, a mere use of multi-path routing schemes could adversely affect the network life as the depletion rate of battery power will be higher. Hence, setting a threshold value is critical to trigger multi-path routing as and when congestion occurs to balance the network life and improve the performance. Measuring only one parameter to ascertain network conditions may present a false indication of network performance. As a result, the measurement of multiple network parameters may provide an enhanced indication of network performance. Moreover, the effect of different traffic loads has been evaluated to completely assess the applicability.

\section{CONGESTION AWARE AND ADAPTIVE DSR ALGORITHM WITH LOAD- BALANCING}

The existing DSR protocol was modified to perform multi-path routing with congestion aware and adaptive feature. In the proposed protocol, the battery level and queue length are used as the key resource utilization parameters.

\subsection{Monitoring Congestion}

Congestion should be defined for effective routing of packets in the network. In the present paper, the congestion was assumed to be in existence when queue length was near capacity or when battery level fell below a pre-defined threshold. The proposed protocol works similar to normal DSR protocol if the current energy level is between 10 to $100 \%$ of initial energy level and queue length is less than 50. The modified DSR algorithm invokes multi-path routing when it exceeds these threshold values.

\subsection{Energy Calculation and Queue Length}

The calculation of energy level is important to determine the battery level of every node during active data transmission. In this work, it was assumed that the battery level of a wireless node decreased when the node initiated data transmission or when the node forward packets. Our proposed algorithm calculates the energy level of a node before forwarding data packet to the next node. The queue length has been considered as another important resource utilization parameter and the best indicator of congestion. In a particular instance, when a data packet needs to wait in queue for longer time, there is a possibility for unexpected delay in transmission or dropping of packets.

\subsection{Load Balancing}

Whenever congestion occurs, the multi-path routing is invoked which will select unequal cost paths stored in the secondary cache. The modified DSR protocol transmits a RERR message when the energy level reach 10 percent and the queue reach their capacity of 50 packets. When the nodes in the downstream receive the RERR message, it enables multi-path routing and searches their primary and secondary caches for multiple routes to the intended destination. During the period of congestion, the route admission control ignores subsequent RREQ messages for 12 seconds, a 2 second more than the default flooding time of RREQ. This is intentionally set for the routes to converge prior to the expiration of this route admission control timer. After the expiration of the route admission control timer, RREQ messages are processed again and the protocol behaves as normal or as multi-path manner depending upon the existence of congestion.

\section{SIMULATION FRAMEWORK 4.1 Simulation Set-up}

The Simulation of the network has been carried out using NS-2 version 2.30 [21-23]. The 802.11 MAC protocol is defined to be the wireless channel. Each mobile node uses a Two-Ray Ground radio propagation model with an Omni antenna. The initial battery capacity of each node assumed to be $100 \%$. The battery capacity of a mobile node was decremented in a predefined manner by the txPower and rxPower levels which remains constant throughout the simulation. In order to conform to the IEEE 802.11b specification, the dataRate_ variable was set to $11 \mathrm{Mbps}$ and the basicRate_ variable was set to $1 \mathrm{Mbps}$. These settings are important to change the default NS-2 setting of $2 \mathrm{Mbps}$. The CTS / RTS process used in IEEE $802.11 \mathrm{~b}$ within NS-2 normally generate huge volumes of data that made processing the trace files complicated. In order to restrict the processing of unnecessary data, the RTSThreshold_ variable was set to 3,000 bytes which restrict the processing of packets that have a size greater than 3,000 bytes. As per the definition, the simulation scenarios did not generate packets larger than 512 bytes which is well below the set 
RTSThreshold_variable. The random topology consists of $800 \mathrm{x}$ 800 meter grid with 20 nodes. The packet sizes of CBR multimedia streams were fixed at 512 bytes with each node had a maximum queue size of 50 packets. The simulation time was set to be 400 seconds and the speed of the node is $10 \mathrm{~m} / \mathrm{s}$. The performance of the network was evaluated at different traffic loads of 10,15 and 20 CBR by keeping the pause time and data rate constant at 4 seconds and 200 PPS, respectively.

\subsection{Performance Metrics}

For streaming multimedia application, throughput alone cannot be considered as performance indicator and that traffic load may have a deceive effect on other parameters. Reide and Seide [24] insisted on measuring packet loss, end-to-end delay and jitter to further validate the network performance. Durkin [25] stated that packet loss and delay are the most important measures for CBR traffic. In the present paper, the performance metrics such as average throughput, average jitter, average end-to-end delay and percentage packet loss was calculated and evaluated for both original DSR and modified DSR using the following relationships.

\subsubsection{Average Throughput}

$$
\text { AverageThroughtput }=\frac{\text { Number of bytes received } \times 8}{\text { Simulation time } \times 1000} \mathrm{kbps}
$$

\subsubsection{Average Jitter}

Jitter is a measure of variation in delay across multiple packets associated with a given traffic flow. In streaming multimedia applications, only a small and limited amount of jitter is tolerable.

\subsubsection{Average end-to-end delay}

The average end-to-end delay is a measure of average time taken to transmit each packet of data from the source to the destination. Network congestion is indicated by higher end-to-end delays.

\section{RESULTS AND DISCUSSION}

\subsection{Average Throughput}

In low traffic load, the throughput of original DSR is $586 \mathrm{kbps}$ as shown in Figure 1. The throughput value should generally increase as traffic load increases. However, in medium traffic load, it increases to $639 \mathrm{kbps}$ and then decreases to $496 \mathrm{kbps}$ at high traffic load. This is indicating the capability of the network in transmitting data up to 15 CBR traffic flow without loss of much data packets. An increasing traffic load beyond that limit leads to loss of more data packets which ultimately result in reduced throughput. The modified DSR shows inferior performance to original DSR and exhibits a decreasing trend in throughput value with increasing traffic load. In low traffic load, the throughput is $544 \mathrm{kbps}$, slightly lower than the original DSR and in medium traffic load the throughput is $200 \mathrm{kbps}$ lower than original DSR. In high traffic load, the throughput is only $358 \mathrm{kbps}$. In a multipath environment, the traffic should get uniformly distributed to achieve better throughput values whenever congestion exists. Among the two congestion parameters, the chances of queue length to exceed the threshold value are higher in this case. However, the increased traffic flow might lead to congestion in intermediate nodes which might make those nodes unavailable for multi-hop routing. This might hinder the possibility of selecting a valid and effective multi-path for data transmission.

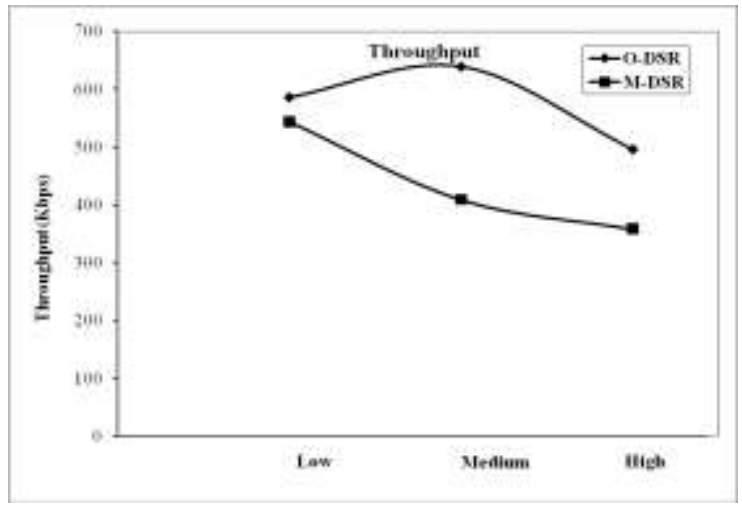

Figure 1. Average throughput of original and modifiedDSR at different workload scenarios.

\subsection{Average Jitter}

The average jitter of modified DSR is marginally higher in low and high traffic load scenarios and is lower in medium traffic load as shown in Figure 2. The increase in jitter values is primarily due to the increase in traffic flow among the multi-path.

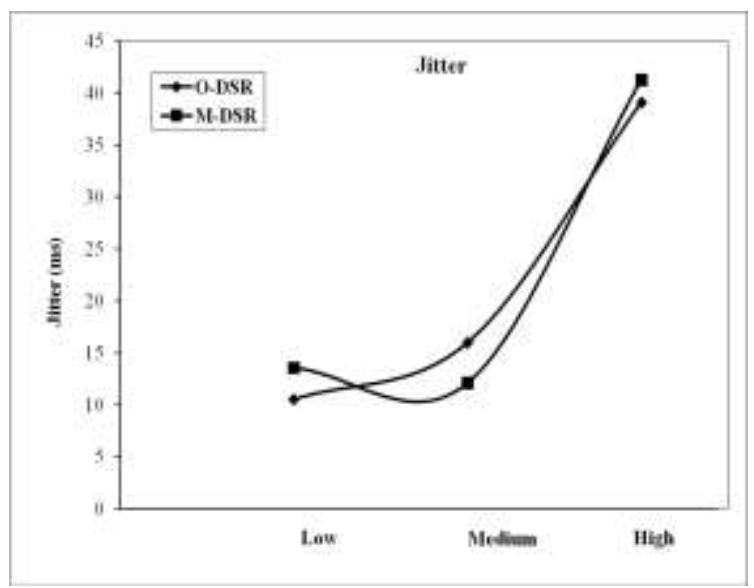

Figure 2. Average jitter of original and modified DSR at different workload scenarios.

\subsection{Average end-to-end delay}

Figure 3 shows the average end-to-end delay of original and modified DSR at low, medium and high traffic load scenarios. The original DSR result in higher end-to-end delays ranging from 587 to $1014 \mathrm{~ms}$. In original DSR, the need for transmitting data over a single path irrespective of traffic flow resulted in drastic increase in delay values. The use of multi-path in distributing the traffic overcomes the problem which exhibit reduced delay values. In different traffic load scenarios, the delay varies between 254 kbps to $619 \mathrm{kbps}$. 


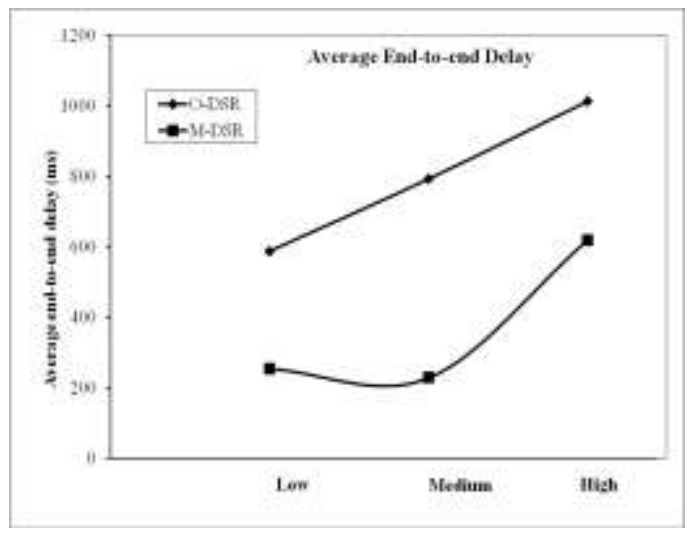

Figure 3. Average end-to-end delay of original and modified DSR at different workload scenarios.

\section{CONCLUSION}

In this paper, congestion aware and adaptive DSR Algorithm with load-balancing for MANET is proposed. In random traffic load scenarios, the throughput of modified DSR is marginally lower compared to original DSR. This is indicating the least influence of multi-path on traffic load. The jitter values also higher for modified DSR, except for the medium traffic load where $4 \mathrm{~ms}$ reduction in jitter was achieved. However, the modified DSR exhibits excellent performance in reducing the end-to-end delay by distributing traffic along multi-path. A huge reduction of 565 $\mathrm{ms}$ in delay was achieved under medium traffic load. The results of the investigation imply that the modified DSR with multi-path routing strategy is a viable alternative to original DSR for streaming multimedia applications in MANET.

\section{REFERENCES}

[1] Macker J M, Corson, Park V. Mobile and wireless internet services: Putting the Pieces together. IEEE Communications Magazine, 2001, 36: 146-155.

[2] Clausen T, Jacquet P. Optimized link state routing protocol. IETF RFC 3626, network Working Group, October 2003.

[3] Parkens C E, Bhagwat P. Highly dynamic DesinationSequenced Distance-Vector Routing (DSDV) for mobile computers. Computer Communications Review, 1994, 24 (4): 234-244.

[4] Perkins C, Belding-Royer E, Das S. Ad hoc on-demand Distance Vector Routing. July 2003, RFC 3561.

[5] Johnson D B, Maltz D A, Broch J. DSR- The dynamic source routing protocol for multi hop wireless ad hoc networks. In Ad hoc Network, Chapter 5, (Perkins C E eds.), Addison-Wesley, 2001.

[6] Corson S, Park V. Temporally-Ordered Routing Algorithm (TORA) Version 1 Functional Specification. Mobile Ad Hoc Network (MANET) Working Group, IETF, Oct. 1999.

[7] Toh C K. A novel distributed routing protocol to support adhoc mobile computing. In Proc. 15th IEEE Annual Int. Phoenix Conference on Computers and Communication. 1996, pp. 480486.

[8] Lei Wang, Lianfang Zhang, Yantai Shu, Miao Dong. Multipath source routing in wireless ad hoc networks. 2000 Canadian
Conference on Electrical and Computer Engineering, 2000,1, pp. 479-483.

[9] Leung R, Jilei Liu, Poon E, Chan A-L C, Baochun Li. MPDSR: a QoS-aware multi-path dynamic source routing protocol for wireless ad-hoc networks. In Proc. 2001 Local Computer Networks, 26th Annual IEEE Conference, 2001, pp. 132-141.

[10] Sung- Ju Lee, Mario Gerla. Dynamic load-aware routing in ad hoc networks. IEEE Conference on Communications, 2001, 10, pp. 3206-3210.

[11] Sung- Ju Lee, Mario Gerla. Split Multi-path Routing with Maximally Disjoint Paths in Ad Hoc Networks. In Proc. Int. IEEE Conf. on Comm., 2001, pp. 3201-3205.

[12] Asis Nasipuri, Robert Castaneda. Performance of Multipath Routing for On-Demand Protocols in Mobile Ad Hoc Networks. Mobile Networks and Applications, 2001, 6: 339-349.

[13] Linifang Zhang, Zenghua Zhao, Yantai Shu, Lei Wang, Yang $\mathrm{O}$ W W. Load balancing of multipath source routing in ad hoc networks. Int. Conference on Communications ICC 2002, 2002, pp. 3197-3201.

[14] Jinglun Shi, Zhang Ling, Shoubin Dong, Zhou Jie. A stability-based multipath routing algorithm for ad hoc networks. In Proc. 14th IEEE Personal, Indoor and Mobile Radio Communications, 2003,1, pp. 516-520.

[15] Zafar Taha, Xian Liu. A model for evaluating multi-route routing in ad hoc networks. Wireless Telecommunications Symposium, 2004, pp. 149-153.

[16] Mueller S, Tsang R P, Ghosal D. Multipath Routing in Mobile Ad Hoc Networks: Issues and Challenges. Invited paper in Lecture Notes in Computer Science, (Maria Carla Calzarossa, Erol Gelenbe eds.), 2004.

[17] Anand Prabhu Subramanian, Anto A J, Janani Vasudevan, Narayanasamy. Multipath Power Sensitive Routing Protocol for Mobile Ad hoc Networks. wireless on-demand network systems. LNCS 2928, 2004, pp. 171-183.

[18] Xiangquan Zheng, Wei Guo, Renting Liu, Yongchun Tian. A New Dynamic Load-aware Based Load-balanced Routing for Ad Hoc Networks. IEEE, 2004, 407-411.

[19] De Rango F, Lonetti P, Marano S. Energy-aware metrics impact on Multi-path DSR in MANETs environment. Int. Symp. on Performance Evaluation of Computer and Telecommunication Systems, 2008, pp. 130-137.

[20] Duc A Tran, Harish Raghavendra. Congestion Adaptive Routing in Mobile Ad Hoc Networks. IEEE transactions on parallel and distributed systems, 2006, 17: 1294-1305.

[21] NS-2 with Wireless and Mobility Extensions, available via web-site http://www.monarch .cs.cmu.edu.

[22] The ns Manual. The VINT Project. A Collaboration between researchers at UC Berkeley, LBL, USC/ISI, and Xerox PARC.December 13, 2003.

[23] The Network Simulator - NS2. Availabl from: http://www.isi.edu/nsnam/ns/, 2007.

[24] Reide N, Seide R. 802.11 (Wi-Fi) Networking Handbook. first ed., CA, McGraw-Hill, 2003.

[25] Durkin. Voice-enabling the data network: H.323, MGCP, SIP, QoS, SLAs, and security. first ed., Indianapolis, IN, Cisco Press, 2003. 